\title{
Fault Analysis in Four-Wire Distribution Networks
}

\author{
Rade M. Ciric ${ }^{1}$, Luis F. Ochoa ${ }^{2}$, Antonio Padilla-Feltrin ${ }^{2}$, Hassan Nouri ${ }^{1}$ \\ e-mail: rciric@netscape.net, luis_ochoa@ieee.org, padilha@dee.feis.unesp.br, hassan.nouri@uwe.ac.uk \\ ${ }^{1}$ University of the West of England, Bristol \\ Faculty of Computing, Engineering and Mathematical Sciences \\ School of Electrical and Computer Engineering \\ Frenchay Campus, Coldharbour Lane \\ Bristol BS16 1QY - UK \\ ${ }^{2}$ UNESP - Universidade Estadual Paulista "Julio de Mesquita Filho" \\ Faculdade de Engenharia de Ilha Solteira \\ Av. Brasil 56 CEP 15385-000 Ilha Solteira - SP - Brazil
}

\begin{abstract}
The neutral wire in most existing power flow and fault analysis software is usually merged into phase wires using Kron's reduction method. In some applications such as fault analysis, fault location, power quality studies, safety analysis, loss analysis, etc., knowledge of the neutral wire and ground currents and voltages could be of particular interest. In this paper, a general short circuit analysis algorithm for three-phase four-wire distribution networks, based on the hybrid compensation method, is presented. In this novel use of the technique, the neutral wire and assumed ground conductor are explicitly represented. A generalized fault analysis method is applied on the distribution network for conditions with and without embedded generation. Results obtained from several case studies on medium and low voltage test networks, with unbalanced loads, for isolated and multi-grounded neutral scenarios, are presented and discussed. Simulation results show the effects of neutrals and system grounding on the operation of the distribution feeders.
\end{abstract}

\section{INTRODUCTION}

Three-phase four-wire distribution networks (DNs) are widely adopted in modern power distribution systems [1], [2], [3], [4], [5] and [6]. A multi-grounded three-phase four-wire network has a higher sensitivity of fault protection than a three-phase three-wire network. The neutral (return) current is due to both the unbalanced load and the non-linear characteristics of electrical equipment through the distribution feeder. The neutral current may be larger than phase currents if three-phase loads are 
noticeably unbalanced in some segments. The neutrals play an important role in power quality and safety problems [6], [7] and [8]. The application of neutrals varies widely from country to country and even from utility to utility within the same country [3].

The neutral wire in existing power flow and fault analysis software is usually merged into phase wires using Kron's reduction method. Since the neutral wire and ground are not represented explicitly, hence the neutral wire and ground currents and voltages remain unknown. In some applications like fault analysis, power quality and safety studies, loss analysis, etc., knowledge of the neutral wire and ground currents and voltages could be of particular interest.

Methods for short circuit analysis in DNs use symmetrical components or phase network representation. The main advantage of conventional symmetrical component methods for fault analysis is that the three sequence matrices are treated separately [2]. The drawback of the above mentioned methods is related to limitations in the modeling of four-wire distribution networks as well as that it cannot be applied in unbalanced and/or un-symmetrical network configurations. However, several efficient methods exist for fault analysis in phase domain [9], [10], [11], [12], and [13]. The drawback is that the neutral wire and ground are not represented explicitly and therefore post fault neutral wire and ground currents and voltages remain unknown.

In order to calculate post fault neutral and ground currents and voltages in DNs, in this research an efficient and robust three-phase backward-forward procedure from [14] and short circuit hybrid compensation method from [13] are generalized. In other words, the $3 \times 3$-network representation is expanded to $5 \times 5$ network representation which consists of three-phase wires, neutral wire and fictitious ground wire [15]. The generalized fault-analysis method can be considered as a general solution for most existing DNs: medium voltage (MV); low voltage (LV); three-wire, four-wire, with (solidly) grounded neutral or isolated neutral wire; and three-wire or single-wire DNs with earth return.

The objective of this work is to demonstrate the feasibility of including the higher order network models such as $4 \times 4,5 \times 5$ and higher, using the hybrid compensation method [14] for short circuit analysis.

\section{Generalized Power Flow Algorithm}

In the three-phase power flow algorithm, each node or line section in the network is numbered by a single index, regardless of the number of phases of the node or line section [15]. Fig. 1 represents line section $l$ between nodes $i$ and $j$ with shunt admittances and loads attached to the nodes.

Based on Fig. 1, a $5 \times 5$ matrix can represent the series impedance $Z_{l}$ of the line section $l$ : 


$$
\left[Z_{l}\right]=\left[\begin{array}{lllll}
Z_{a a} & Z_{a b} & Z_{a c} & Z_{a n} & Z_{a g} \\
Z_{a b} & Z_{b b} & Z_{b c} & Z_{b n} & Z_{b g} \\
Z_{a c} & Z_{b c} & Z_{c c} & Z_{c n} & Z_{c g} \\
Z_{a n} & Z_{b n} & Z_{c n} & Z_{n n} & Z_{n g} \\
Z_{a g} & Z_{b g} & Z_{c g} & Z_{n g} & Z_{g g}
\end{array}\right] \quad \begin{aligned}
& \text { where } \\
& \text { a }
\end{aligned}
$$

The equations for calculation of 5x5 impedance matrix elements are given in the Appendix of [15]. If any phase, neutral wire or grounding of the line section does not exist, the corresponding row and column in this matrix contain all zero entries. The shunt capacitance part of LV lines can be neglected, except for long runs of lines. The branch numbering scheme for a radial DN is similar to that discussed in [14]. Model of the three-phase four-wire multi-grounded distribution line is shown on Fig. 2.

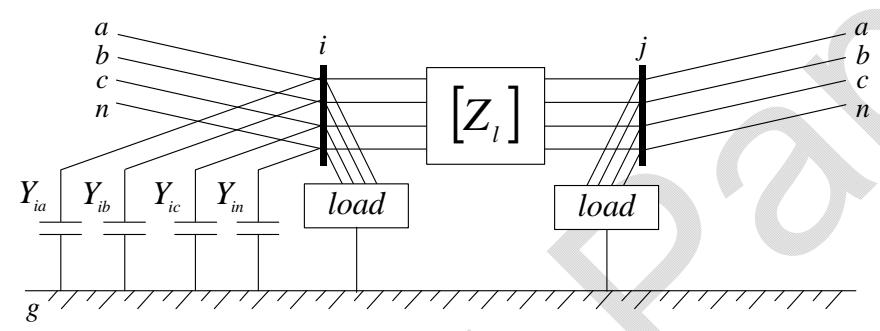

Fig. 1. A three-phase four-wire line section, considering ground

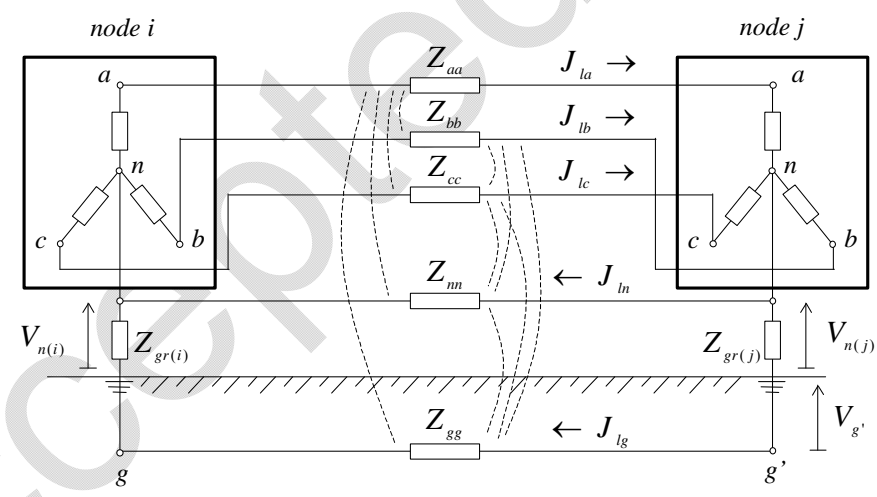

Fig. 2. Model of the three-phase four-wire multi-grounded distribution line.

The power flow algorithm used in this work follows the backward-forward technique described in [15] that is elaborated for the $5 \times 5$ impedance matrix network representation of Fig. 1 . If one assumes the root node to be the slack node and generalizes the procedure of power flow discussed in [14], then the proposed $5 \times 5$ iterative algorithm can be applied to any radial system, provided that the following three steps and conditions are considered.

At iteration $k$ :

Step 1: Nodal current calculation 


$$
\begin{aligned}
& {\left[\begin{array}{c}
I_{i a} \\
I_{i b} \\
I_{i c} \\
I_{i n} \\
I_{i g}
\end{array}\right]^{(k)}=\left[\begin{array}{c}
\left(S_{i a} / V_{i a}\right)^{(k-1)^{*}} \\
\left(S_{i b} / V_{i b}\right)^{(k-1)^{*}} \\
\left(S_{i c} / V_{i c}\right)^{(k-1)^{*}} \\
-\frac{Z_{g i}}{Z_{n n i}+Z_{g i}}\left(I_{i a}^{(k)}+I_{i b}^{(k)}+I_{i c}^{(k)}\right) \\
-\frac{Z_{n n i}}{Z_{n n i}+Z_{g i}}\left(I_{i a}^{(k)}+I_{i b}^{(k)}+I_{i c}^{(k)}\right)
\end{array}\right]} \\
& -\left[\begin{array}{ccccc}
Y_{i a} & & & & \\
& Y_{i b} & & & \\
& & Y_{i c} & & \\
& & & Y_{i n} & \\
& & & & 0
\end{array}\right]\left[\begin{array}{l}
V_{i a} \\
V_{i b} \\
V_{i c} \\
V_{i n} \\
V_{i g}
\end{array}\right]^{(k-1)}
\end{aligned}
$$

where
$I_{i a}, I_{i b}, I_{i c}, I_{i n}, I_{i g}$
are current injections at node $i$
$S_{i a}, S_{i b}, S_{i c}$
are scheduled (known) power injections at node $i$;
$V_{i a}, V_{i b}, V_{i c}, V_{i n}, V_{i g}$
are voltages at node $i$
$Y_{i a}, Y_{i b}, Y_{i c}, Y_{i n}$
are admittances of all shunt elements at node $i$; and
$Z_{g r i}$
is grounding impedance at node $i\left(Z_{g i}=Z_{g r i}+Z_{g g i}\right)$.

Step 2: Backward sweep - section current calculation

Starting from the line section in the last layer and moving towards the root node, the current in line section $l$ is:

$$
\left[\begin{array}{l}
J_{l a} \\
J_{l b} \\
J_{l c} \\
J_{l n} \\
J_{l g}
\end{array}\right]^{(k)}=-\left[\begin{array}{c}
I_{j a} \\
I_{j b} \\
I_{j c} \\
I_{j n} \\
I_{j g}
\end{array}\right]^{(k)}+\sum_{m \in M}\left[\begin{array}{l}
J_{m a} \\
J_{m b} \\
J_{m c} \\
J_{m n} \\
J_{m g}
\end{array}\right]^{(k)}
$$

where

$J_{l a}, J_{l b}, J_{l c}, J_{l n}, J_{l g}$

$M$ are the current flows on line section $l$; and

is the set of line sections connected downstream to node $j$.

Step 3: Forward sweep - nodal voltage calculation

Starting from the first layer and moving towards the last layer, the voltage at node $j$ is expressed as:

$$
\left[\begin{array}{c}
V_{j a} \\
V_{j b} \\
V_{j c} \\
V_{j n} \\
V_{j g}
\end{array}\right]^{(k)}=\left[\begin{array}{c}
V_{i a} \\
V_{i b} \\
V_{i c} \\
V_{i n} \\
V_{i g}
\end{array}\right]^{(k)}-\left[\begin{array}{lllll}
Z_{a a} & Z_{a b} & Z_{a c} & Z_{a n} & Z_{a g} \\
Z_{a b} & Z_{b b} & Z_{b c} & Z_{b n} & Z_{b g} \\
Z_{a c} & Z_{b c} & Z_{c c} & Z_{c n} & Z_{c g} \\
Z_{a n} & Z_{b n} & Z_{c n} & Z_{n n} & Z_{n g} \\
Z_{a g} & Z_{b g} & Z_{c g} & Z_{n g} & Z_{g g}
\end{array}\right]\left[\begin{array}{l}
J_{l a} \\
J_{l b} \\
J_{l c} \\
J_{l n} \\
J_{l g}
\end{array}\right]^{(k)}
$$

\section{Condition 1: Voltage correction}


Voltage correction in the nodes with grounded neutral and downstream nodes should be performed. Voltage in the nodes with neutral grounded at iteration $k$ is as follows:

$$
V_{i n}^{(k)}=Z_{g r i} J_{l g}^{(k)}, i \in\{\text { Grounded nodes }\}
$$

\section{Condition 2: Convergence criterion}

After these steps are executed in one iteration, the power mismatches at each node for all phases, and neutral wire and ground are calculated:

$$
\begin{aligned}
& \Delta S_{i a}^{(k)}=V_{i a}^{(k)}\left(I_{i a}^{(k)}\right)^{*}-Y_{i a}^{*}\left|V_{i a}^{(k)}\right|^{2}-S_{i a} \\
& \Delta S_{i b}^{(k)}=V_{i b}^{(k)}\left(I_{i b}^{(k)}\right)^{*}-Y_{i b}^{*}\left|V_{i b}^{(k)}\right|^{2}-S_{i b} \\
& \Delta S_{i c}^{(k)}=V_{i c}^{(k)}\left(I_{i c}^{(k)}\right)^{*}-Y_{i c}^{*}\left|V_{i c}^{(k)}\right|^{2}-S_{i c} \\
& \Delta S_{i n}^{(k)}=V_{i n}^{(k)}\left(I_{i n}^{(k)}\right)^{*}-Y_{i n}^{*}\left|V_{i n}^{(k)}\right|^{2} \\
& \Delta S_{i g}^{(k)}=V_{i g}^{(k)}\left(I_{i g}^{(k)}\right)^{*}
\end{aligned}
$$

If the real or imaginary part of any power mismatch is greater than a convergence criterion, steps 1,2 and 3 are repeated until convergence is achieved.

\section{Condition 3: Flat start}

The initial voltage for all nodes should be equal to the root node voltage:

$$
\left[\begin{array}{c}
V_{i a} \\
V_{i b} \\
V_{i c} \\
V_{i n} \\
V_{i g}
\end{array}\right]^{(0)}=\left[\begin{array}{c}
V_{r e f} \\
a^{2} \cdot V_{r e f} \\
a \cdot V_{r e f} \\
0 \\
0
\end{array}\right], \quad a=e^{j \frac{2 \pi}{3}} \text {. }
$$

Off-diagonal admittances in (1) are ignored. However their impact on the accuracy of the power flow solution is very small (about $0.1 \%$ [15]). It should be noted that loads in different buses may be grounded with different resistance meaning different voltages $V_{n i}$ and $V_{n j}$. This fact is considered by applying (4) from condition 1 . That is one of the reasons for using $5 \times 5$ network representation in modeling distribution network with multi-grounded neutral wire. Voltages in (1) and (3) have the same reference of zero potential, which is the "remote earth". The concept of "remote earth" is based on model of Carson's line and explained in detail in [16].

Most of the existing models of distribution system elements (lines, loads, shunt capacitors, distribution transformers, embedded generators) can be applied to the generalized power flow method according to the general form given in [17].

\section{ShORT CIRCUIT ANALYSIS}

In order to investigate the effects of neutral wire and grounding in the case of faults, the generalized 
power flow algorithm from [15] is applied in the hybrid compensation short circuit method [13]. The hybrid compensation method combines three basic compensations, i.e. loop break-point compensation, embedded generation compensation, and faults compensation. The hybrid Thevenin equivalent is calculated from (7):

$$
\left[Z_{t}\right] \cdot\left[I_{t}\right]=\left[V_{t}\right]
$$

The diagonal elements of the hybrid Thevenin equivalent matrix are equal to the sum of line section impedance between the compensation nodes (i.e. break points, generator nodes, or fault nodes) and the root node. If two faulted nodes $i$ and $j$ share any common path to the root node, then $Z_{i j}$ is equal to the sum of all line section impedance matrices on the common path. The right-hand-side of (7) represents the voltage mismatch vector for break-point nodes, generator nodes or fault nodes.

The generalized method for fault analysis uses the power flow solution in a 5x5-network representation as a pre-fault condition, and generates post-fault state after updating hybrid current injections. To update hybrid current injections, (7) needs to be solved by current injections. In this paper, only the generalization of compensation for faults will be explained. However the same concept holds for compensation of loops and generators.

General fault boundary conditions in $5 \times 5$ network representations for calculating different types of fault are given in (8):

$$
\left[\begin{array}{c}
V_{t a} \\
V_{t b} \\
V_{t c} \\
V_{t n} \\
V_{t g}
\end{array}\right]=\left[\begin{array}{c}
V_{f a} \\
V_{f b} \\
V_{f c} \\
V_{f n} \\
V_{f g}
\end{array}\right]^{(s)}-\left[\begin{array}{c}
V_{f a} \\
V_{f b} \\
V_{f c} \\
V_{f n} \\
V_{f g}
\end{array}\right]^{(o)}
$$

where

$V_{t}$

is the voltage mismatch vector from (7),

$V_{f}^{(s)}$

is the scheduled voltage (fault boundary condition), and

$V_{f}^{(o)}$ is the pre-fault voltage at the faulted node.

In (8), boundary conditions are given regarding known voltages at the fault points. The problem in modeling neutral wire and ground is that neutral and ground voltages at the fault point are not known. That is why in the expanded short circuit compensation algorithm, the hybrid Thevenin compensation impedance matrix $Z_{t}$ is determined in the form of a $3 \times 3$-network representation. The physical explanation of this concept is as follows. In case of a fault in the distribution network, the fault point is fed by the substation and embedded generator (which are both sources of fault currents) by injecting currents 
through only the phase wires which are named as the "active wires". However, the neutral wire and fictitious ground conductor stay "passive" since they cannot feed the fault point. According to the above analysis, one can assume that representation of the hybrid Thevenin compensation impedance matrix $Z_{t}$ for this study in the form of $3 \times 3$ network representation is justified. Hence, boundary fault conditions for $V_{f}^{(s)}$ are kept the same as for the original hybrid compensation method [13], namely in $3 \times 3$ notation.

Following the original hybrid compensation method [13], after solving fault currents $I_{t}$ by (7), node current injections of phases $a, b$ and $c$ are updated by adding post-fault current injections with pre-fault node current injections. The pre-fault node current injections of phases $a, b$ and $c$ are determined from power flow solution.

For three-line-to-ground faults, at a three-phase line section, post-fault current injections are given by (9),

$$
\left[\begin{array}{l}
I_{f a}{ }^{(p)} \\
I_{f b}(p) \\
I_{f c}(p)
\end{array}\right]=\left[\begin{array}{l}
I_{t a} \\
I_{t b} \\
I_{t c}
\end{array}\right]
$$

where

$I_{f a}^{(p)}, I_{f b}{ }^{(p)}, I_{f c}^{(p)}$ are post-fault phase current injections that will be added to faulted nodes;

$I_{t a}, I_{t b}, I_{t c}$ are fault currents obtained by solving (7);

For double-line-to-ground faults or line-to-line faults, on phases $b$ and $c$, post-fault current injections $I_{f}^{(p)}$ can be formed by (10):

$$
\left[\begin{array}{l}
I_{f a}{ }^{(p)} \\
I_{f b}{ }^{(p)} \\
I_{f c}(p)
\end{array}\right]=\left[\begin{array}{l}
0 \\
I_{t b} \\
I_{t c}
\end{array}\right]
$$

For single-line-to-ground fault on phase $a$, post-fault current injection $I_{f}^{(p)}$ is determined from (11):

$$
\left[\begin{array}{l}
I_{f a}{ }^{(p)} \\
I_{f b}{ }^{(p)} \\
I_{f c}(p)
\end{array}\right]=\left[\begin{array}{l}
I_{t a} \\
0 \\
0
\end{array}\right]
$$

Post-fault node current injections of the neutral wire and ground are then calculated using (12), 


$$
\begin{aligned}
& I_{f n}^{(p)}=-\frac{Z_{g f}}{Z_{n n f}+Z_{g f}}\left(I_{f a}^{(p)}+I_{f b}^{(p)}+I_{f c}^{(p)}\right) \\
& I_{f g}^{(p)}=-\frac{Z_{n n f}}{Z_{n n f}+Z_{g f}}\left(I_{f a}^{(p)}+I_{f b}^{(p)}+I_{f c}^{(p)}\right)
\end{aligned}
$$

where

$$
\begin{aligned}
& I_{f n}{ }^{(p)}, I_{f g}{ }^{(p)} \\
& Z_{g r f} \\
& Z_{n n f}, Z_{g g f}
\end{aligned}
$$$$
\text { are post-fault neutral wire and ground current injections, }
$$$$
\text { is the grounding impedance at the faulted node, and }
$$
are neutral wire and fictitious ground conductor impedances in the faulted

$$
\operatorname{section}\left(Z_{g f}=Z_{g r f}+Z_{g g f}\right) \text {. }
$$

Equation (12) is the consequence of the Kirchoff's current law (current divider), Fig. 2. The mutual coupling between phase lines, neutral wire and ground is taken into account through voltage equation (3). Once the post-fault current injections in all simultaneously faulted nodes are obtained, post-fault line section currents and node voltages are calculated using a single iteration of the power flow method, (1) to (6). With this approach, compensation for a fault is expanded to $5 \times 5$ impedance matrix network representation. The same concept holds for the compensation of loops and generators, where the hybrid Thevenin compensation impedance matrix $Z_{t}$ is determined by considering the $3 \times 3$ impedance matrix network representation.

The flow chart of the generalized hybrid compensation short circuit method is shown in Fig. 3.

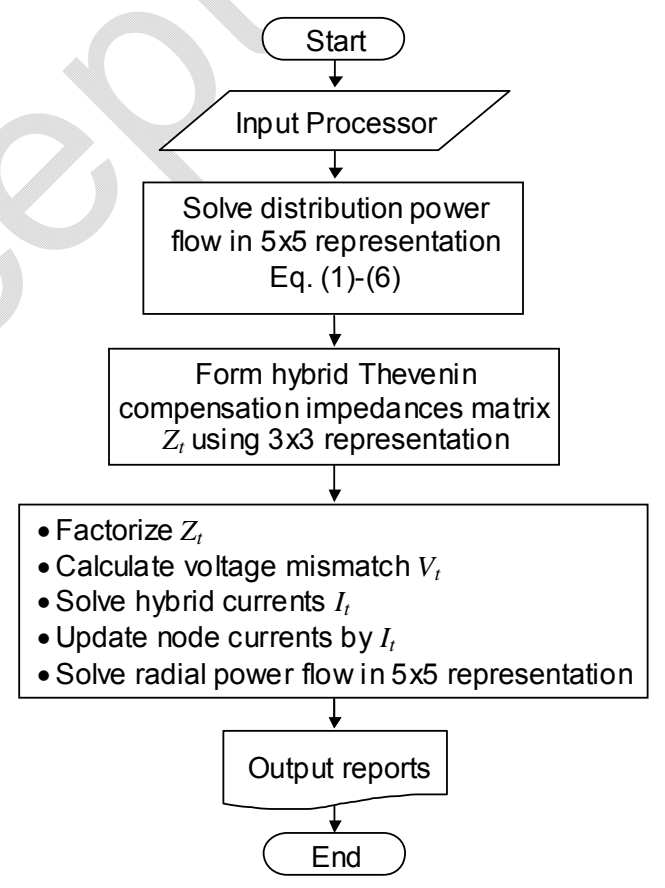

Fig. 3. Flow chart of the generalized hybrid compensation short circuit method. 


\section{TEST NETWORKS}

The generalized hybrid compensation method for short circuit analysis is applied on several MV and LV distribution networks. In this paper the following networks are used:

LV-29: 29-bus real-life four-wire three-phase LV feeder from Braganca Paulista city, SP-Brazil (data supplied by Grupo Rede) (Fig. 4) [15]. The total demand is $17.7 \mathrm{~kW}$, and the loads are distributed along the network (the most distant node is $170 \mathrm{~m}$ from the root node). Base voltage is $V b=210.0 \mathrm{~V} ;$ and

IEEE-34: 34-bus IEEE four-wire three-phase MV feeder (Fig. 5) [15] and [18]. The total demand is $1770.0 \mathrm{~kW}$, and $72 \%$ of the loads are concentrated $56 \mathrm{~km}$ far away from the root node (the most distant node is $59 \mathrm{~km}$ from the substation). Base voltage is $V b=24.9 \mathrm{kV}$ and reference voltage in the root node is $V_{\text {ref }}=25.647 \mathrm{kV}$.

Networks with isolated and multi-grounded neutral wires are considered. The load data for LV-29 test network is given in [15]. Simplifying, the transformer $24.9 / 4.16 \mathrm{kV} / \mathrm{kV}$ in the original IEEE-34 test feeder is replaced with a line and the network is modeled with single voltage level. The automatic voltage regulator is also not represented.

The four-wire model impedances are calculated considering a ground resistivity of $100 \Omega-\mathrm{m}$ and using the formulation shown in the Appendix of [15].

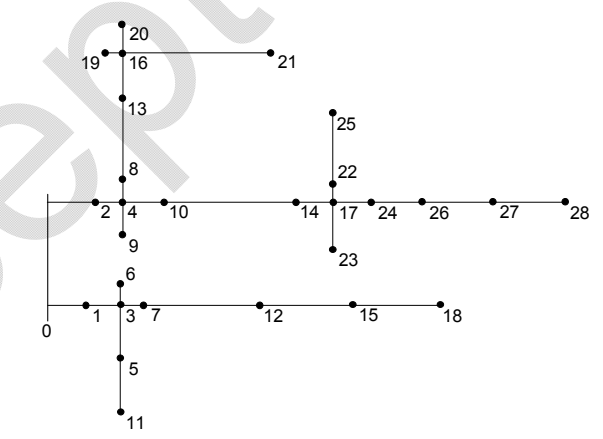

Fig. 4. LV-29 test feeder.

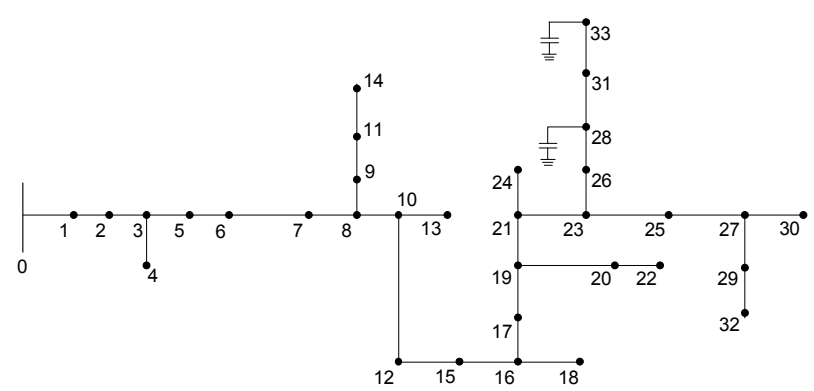

Fig. 5. IEEE-34 bus test feeder. 


\section{Applications: SOME Results}

The expanded hybrid compensation short circuit method is tested using radial MV and LV DNs considering constant admittance load modeling. Distribution LV network with multi-grounded neutrals, as well as MV network with isolated and multi-grounded neutrals are considered. Cases with and without embedded generators in the MV DN are also considered. For simplicity, the impedance at the point of fault for all cases is considered to be zero. It is known that transformer configurations may result in different post-fault current [19]. This paper does not consider various transformer configurations. However, most of the existing transformer configurations can be introduced in the expanded short circuit algorithms according to the transformer models presented in [17] and [19].

Fig. 6 shows a profile of the pre-fault neutral wire and ground currents in the LV-29 DN for a multigrounded neutral (nodes 4, 11, 18, 20, 28 with $R_{g r}=1.0 \Omega$ ). An inspection of Fig. 6 suggests that current in the neutral wire in the head of the feeder before the fault is $33 \%$ of the phase currents (average of 26.7

A) and the return current through the ground before the fault is $0.2 \%$ of the phase currents.

Results of fault analysis applied on the LV-29 DN are shown on Fig. 7 and Fig. 8. The equivalent impedance of the distribution system at the substation $\mathrm{MV} / \mathrm{LV}$ is set to $R_{\text {sys }}=1.0 \Omega /$ phase. Fig. 7 shows post-fault neutral and ground voltage at node 27 for different types of fault. Analysis of Fig. 7 exhibits the largest voltage drop in the neutral wire and ground for a line-to-ground fault, while the smallest voltage drop appears in the neutral wire and ground in the case of a line-to-line fault.

Fig. 8 shows post-fault neutral wire and ground currents in the head of the feeder (node 2) for different types of fault which determine the magnitude of the current. A higher current relates to the most unsymmetrical fault whereas a lower current to symmetrical faults. As shown in Fig. 8, the return current (90 A) through the neutral at node 2 is $46 \%$ the current capacity of the neutral conductor in that section. Referring to Fig. 7, voltage of the neutral wire at node $27(12.6 \mathrm{~V})$ is caused by the flow of return current in the neutral conductor and high ground impedance (resistance) which could be due to lack of or difficulty in implementing a proper grounding. For such cases most of the current flows into the neutral conductor as opposed to some flowing into the ground. High neutral voltage at the end node may not only cause common-mode noise problems in sensitive electronic equipment but it may also represent a safety issue [4]. 


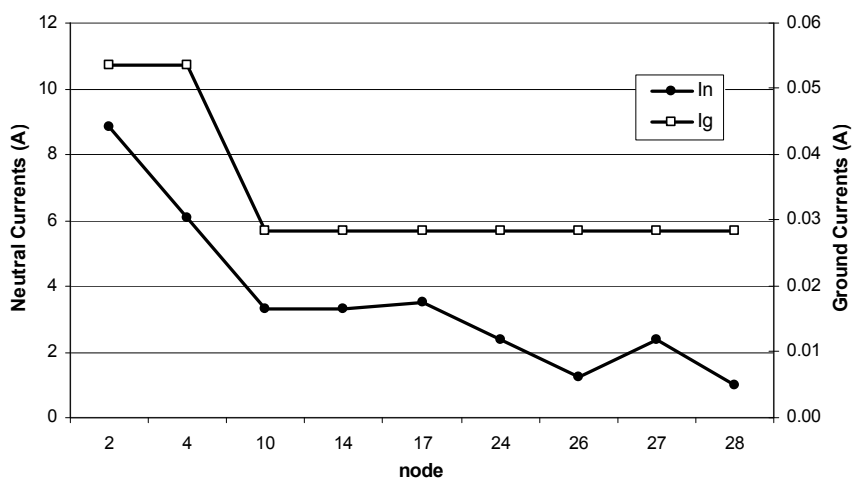

Fig.6. Pre-fault Neutral and Ground Currents; LV-29 multi-grounded neutral.

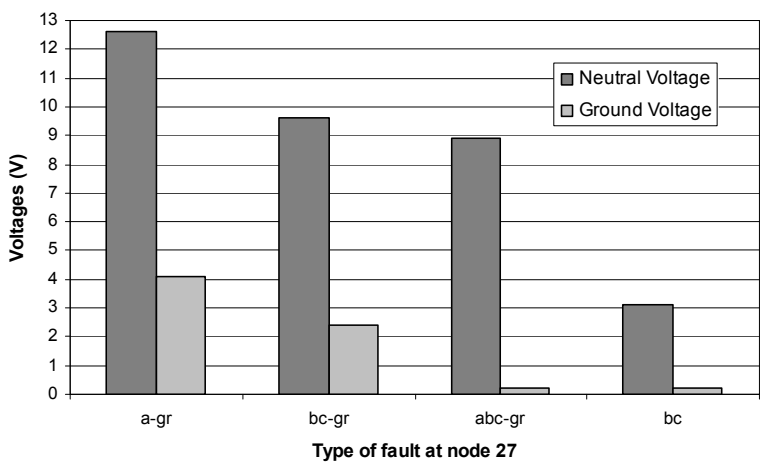

Fig. 7. Neutral and Ground Voltages for different types of fault at node 27; LV-29 multi-grounded neutral.

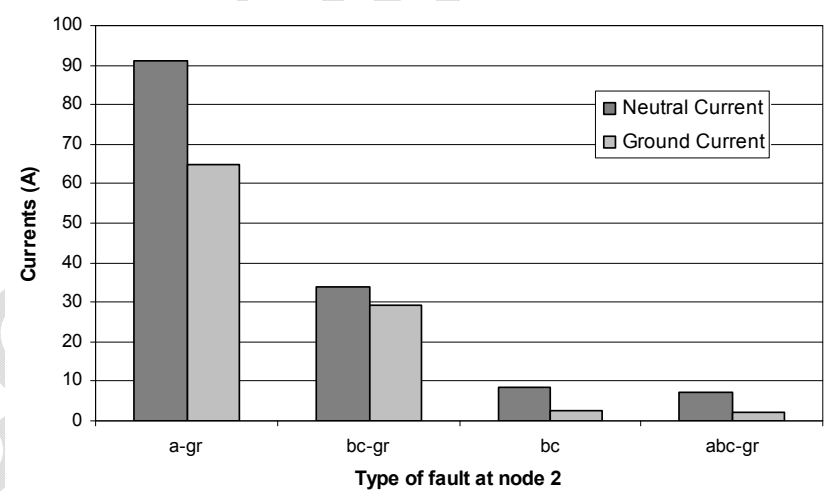

Fig. 8. Neutral and Ground Currents at node 2 for different types of fault; LV-29 multi-grounded neutral.

Results of the fault analysis applied on the IEEE-34 bus DN with isolated and multi-grounded neutral wires are shown in Fig. 9 to Fig. 12. Also considered is analysis of the network for conditions with and without embedded generation at node 23. The embedded generator node is modeled as a PV node. For this study, the real generator power is $P_{\text {gen }}=900 \mathrm{~kW}$, set generator voltage is $V_{\text {gen }}=24.5 \mathrm{kV}$, and calculated generator injected reactive power is $Q_{g e n}=120 \mathrm{kVAr}$. The internal impedance of the generator is $Z_{g e n}=(0.6+\mathrm{j} 1.5) \Omega /$ phase and used system equivalent impedance at the substation $\mathrm{HV} / \mathrm{MV}(\Delta / \mathrm{grounded}$ $\mathrm{Y})$ is $Z_{\text {sys }}=(5+\mathrm{j} 5) \Omega /$ phase. 
Fig. 9 shows post-fault voltages for the IEEE-34 bus DN with isolated neutrals, without embedded generators, for a double-line-to-ground fault (phases $b$ and $c$ to ground) at node 10 . The post-fault neutral voltage in the end node is $8.9 \mathrm{kV}$, and ground voltage is $2 \mathrm{kV}$, which represents $72 \%$ and $16 \%$ of the pre-fault phase voltages $(12.3 \mathrm{kV})$, respectively.

Fig. 10 and Fig. 11 show post-fault impact of the generator on the state of the considered DN with multi-grounded neutral wires. The post-fault current in phase $a$ as well as through the ground (node 1), are increased by a factor of two as opposed to current through the neutral wire that is reduced by approximately $40 \%$.

Fig. 12 shows characteristics of the neutral and ground currents for a three-line-to-ground fault at different nodes for conditions where an embedded generator is connected to node 23 and the network has a multi-grounded neutral wire scheme. Fig. 12 suggests that the magnitude of the neutral and ground currents are inversely proportional to the distance of the fault from the substation. The inclusion of the embedded generator significantly increases neutral and ground currents after the fault at the generator node and the neighboring nodes.

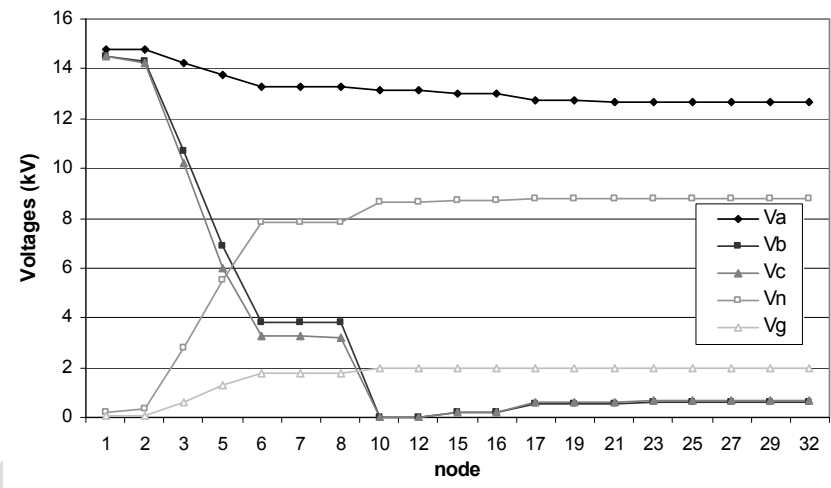

Fig. 9. Post Fault voltage characteristics of a double-line-to-ground fault (phases b and c) at node 10 on the IEEE-34 bus feeder with isolated neutral and without a generator.

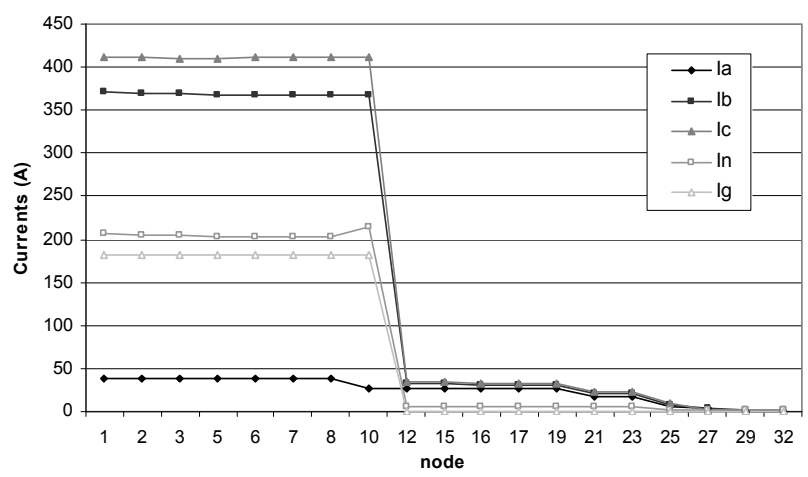

Fig. 10. Phase currents profiles at various nodes for a double-line-to-ground (phase b and c) fault at node 10 on the IEEE-34 bus feeder with multi-grounded neutral and without a generator. 


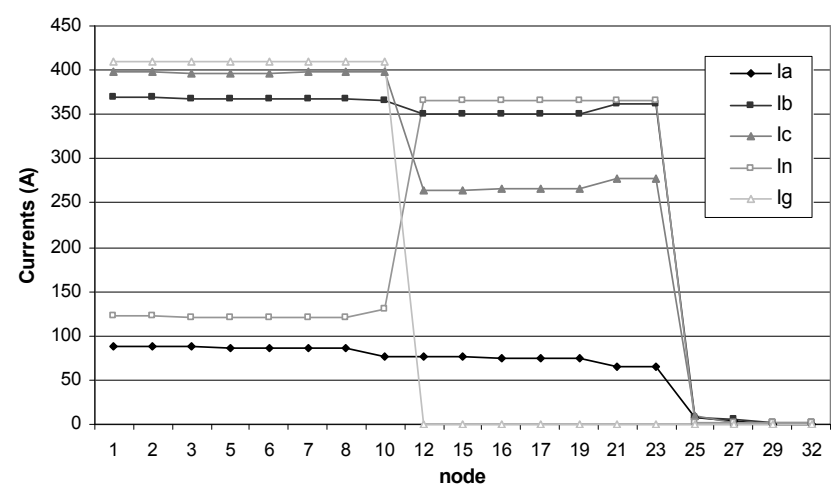

Fig. 11. Phase Currents profile at various nodes for a double-line-to-ground (phases b and c) fault at node 10 on the IEEE-34 bus feeder with multi-grounded neutral and with the generator at node 23 .

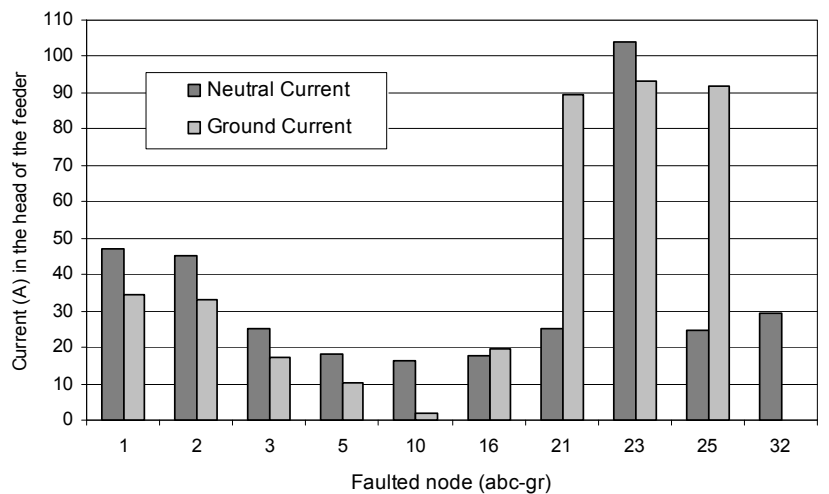

Fig. 12. Neutral and Ground Currents for three-lines-to-ground fault at different nodes on the IEEE-34 bus feeder with multi-grounded neutral and with generator at node 23.

Results obtained with the expanded hybrid compensation method are compared with those obtained using the conventional symmetrical component short circuit method [2]. The accuracy in all considered cases is in the order of $3 \%$.

The following insights are drawn from the performed simulations:

- High-order line models such as $4 \times 4,5 \times 5$ and higher may be included in a common solution method for a three-phase hybrid compensation algorithm for fault analysis [13] with only minor changes;

- Kron's reduction method in power flow and fault analysis is justified when neutral and ground current and voltages are not of specific interest [15];

- Post-fault neutral and ground voltages and currents in real-life DNs may have significant values and may represent power quality and safety issues;

- The embedded generator in the distribution network significantly affects the neutral and ground voltages and currents after a fault; 
- The generalized methodology for fault analysis can be applied on the experimental five-wire, threephase DN, proposed in [5] since the fourth-grounding wire and the fifth-neutral wire can be represented explicitly.

\section{CONCLUSION}

In this paper a method for fault analysis in four-wire DNs, is discussed. The methodology for fault analysis is based on the generalized backward-forward technique [15] and hybrid compensation short circuit method [13]. The used hybrid compensation short circuit method has not lost its efficiency, while neutral wire and ground are represented explicitly. The methodology is of general usage since it enables fault analysis in most of the existing DNs (LV, MV, three-wire, four-wire with isolated/grounded neutral wire, etc.).

Simulation results show the effects of neutrals and system grounding on the operation of MV and LV DNs. Results show that post-fault neutral and ground voltages and currents in real-life DNs may have significant values therefore should be considered in safety analysis.

The software package based on the generalized hybrid compensation short circuit method, presented in this work, can be an efficient tool for fault analysis (fault detection and localization), relay settings, power quality studies and safety analysis, where neutral wire and ground currents and voltages are of particular interest.

\section{REFERENCES}

[1] LAKERVI, E. and HOLMES, E. J.: 'Electricity Distribution Network Design' (IEE Power Engineering Series 21, London: Peter Peregrines Ltd. On behalf of the IEE, 1995)

[2] ANDERSON, P. M.: 'Analysis of Faulted Power Systems' (IEEE Press Power Systems Engineering Series, New York, pp. 71-83, 1995)

[3] Meliopoulos, A. P., KENNEDy, J., NUCCI, C. A., BORGHETTI, A., and CONTAXIES, G.: 'Power distribution practices in USA and Europe: Impact on power quality'. 1998 International Conference on Harmonics and Quality of Power, 1998, pp. $24-29$

[4] CHEN, T. H., and YANG, W. C.: 'Analysis of multi-grounded four-wire distribution systems considering the neutral grounding', IEEE Trans. Power Delivery, 2001, 16 (4) pp. 710-717

[5] SHORT, T. A., STEWART, J., SMITH, D. R., O’BRIEN, J., and HAMPTON, K.: 'Five-wire distribution system demonstration project', IEEE Trans. Power Delivery, 2002, 17 (2) pp. 649-654 
[6] BALDA, J. C., OLIVA, A. R., MCNABB, D. W., and RICHARDSON, R. D.: 'Measurements of neutral currents and voltages on a distribution feeder', IEEE Trans. Power Delivery, 1997, 12 (4) pp. $1799-1804$

[7] DAS, J. C., and OSMAN, R. H.: 'Grounding of AC and DC low-voltage and medium-voltage drive system', IEEE Trans. Industry Applications, 1998, 34 (1) pp. 205-216

[8] 'IEEE Recommended Practice for Grounding of Industrial and Commercial Power Systems'. IEEE Standard 142-1991, 1992

[9] CHEN, T. H., CHEN, M. S., LEE, W. J., KOTAS, P., and OLINDA, P. V.: 'Distribution system short circuit analysis - a rigid approach', IEEE Trans. on Power Systems, 1992, 7 (1) pp. 444-450

[10]GROSS, G., and HONG, H. W.: 'A two step compensation method for solving short circuit problems', IEEE Trans. on Power Apparatus and Systems, 1982, PAS-101 (6) pp. 1322-1331

[11] ALVARADO, F., MONG, S., and ENNS, M.: 'A fault program with macros, monitors and direct compensation in mutual groups', IEEE Trans. on Power Apparatus and Systems, 1985, PAS-104 (5) pp. 1109-1120

[12]BRANDWAJN, V., and TINNEY, W. F.: 'Generalised method of fault analysis', IEEE Trans. on Power Apparatus and Systems, 1985, PAS-104 (6), pp. 1301-1306

[13]ZHANG, X., SOUDI, F., SHIRMOHAMMADI, D., and CHENG, C.: 'A distribution short circuit analysis approach using hybrid compensation method', IEEE Trans on Power Systems, 1995, 10 (4), pp. 2053-2059

[14]CHENG C. S., and SHIRMOHAMMADI, D., 'A three-phase power flow method for real-time distribution system analysis', IEEE Trans on Power Systems, 1995, 10 (2), pp. 671-769

[15]CIRIC, R. M., PADILHA, A., and OCHOA, L. F.: 'Power Flow in Four-Wire Distribution Networks-General Approach', IEEE Trans on Power Systems, 2003, 18 (4), pp. 1283-1290

[16] CIRIC, R. M., OCHOA, L. F., and PADILHA, A.: 'Power flow in distribution networks with earth return', International Journal of Electrical Power and Energy Systems, 2004, 26, pp. 373-380

[17]ZHU, Y., and TOMSOVIC, K.: 'Adaptive power flow method for distribution systems with dispersed generation', IEEE Trans. Power Delivery, 2002, 17 (3), pp. 822-827

[18] KERSTING, W. H.: 'Radial distribution test feeders', IEEE Trans. on Power Systems, 1991, 6 (3), pp. $975-985$

[19]TAN, A., LIN, W. H. E., and SHIRMOHAMMADI, D.: 'Transformer and load modeling in short circuit analysis for distribution systems', IEEE Trans. on Power Systems, 1997, 12 (3), pp. 13151322 